\title{
Surgical Control of a Primary Hepatic Carcinoid Tumor: A Case Report
}

\author{
Norio Yokoigawa ${ }^{a}$ Hiroaki Kitade $^{a}$ Masaharu Ohishi ${ }^{a}$ \\ Takashi Ozaki $^{\mathrm{a}}$ Takeshi Miyaso $^{\mathrm{a}}$ Satoshi Okazaki ${ }^{\mathrm{a}}$ \\ Koji Nakai ${ }^{a}$ Ryo Yoshida ${ }^{a}$ Seiki Imada ${ }^{a}$ Atsushi Imamura ${ }^{a}$ \\ Yusai Kawaguchia Hideho Takada ${ }^{a}$ Nobuaki Shikata $^{b}$ \\ Toshihito Sekic A.-H. Kwon ${ }^{\mathrm{a}}$ \\ Departments of aSurgery, bPathology II, and ' Gastroenterology and Hepatology, \\ Kansai Medical University, Osaka, Japan
}

\section{Key Words}

Primary $\cdot$ Carcinoid tumor $\cdot$ Liver

\begin{abstract}
We report a primary hepatic carcinoid tumor occurring in a 47-year-old man. The patient consulted our hospital complaining of epigastralgia. Abdominal ultrasonography, computed tomography scanning, and magnetic resonance imaging showed a large mass in the right lobe of the liver. FDG-PET revealed 18F-FDG uptake by the right hepatic lobe. The tumor was a solid mass with cystic components, approximately $15 \mathrm{~cm}$ in diameter. We conducted an extended right lobectomy of the liver. The resected specimen was a solid tumor with cystic components and hemorrhagic lesion. Microscopic findings showed that the tumor cells had round nuclei and formed trabecular patterns. Immunohistologically, tumor cells were stained positive for chromogranin A, neuron specific enolase, CD56, and S-100. Careful examinations before and after the operation revealed no other possible origin of the tumor. Based on these findings, the tumor was diagnosed as a primary hepatic carcinoid. This is a report of a rare case of a primary hepatic carcinoid tumor with a discussion of several other relevant reports.
\end{abstract}

\section{Introduction}

Carcinoid tumors were first reported by Oberndorfer in 1907 [1]. They may occur in a variety of locations throughout the body and often metastasize to the liver. However, primary hepatic carcinoid tumor (PHCT) is very rare and, to our knowledge, only 90 cases have been reported. The diagnosis of PHCT is based principally on the 
histopathological confirmation of carcinoid tumor, and it is necessary to identify the absence of another primary site. We report a case of PHCT and describe the clinical features.

\section{Case Report}

The patient was a 47-year-old man who complained initially of epigastralgia and of a palpable abdominal mass. There was no history of hematemesis, jaundice, vomiting, flushing or diarrhea. Although hemangioma of the liver had been noticed 10 years previously, the patient did not visit the hospital thereafter. Laboratory data showed normal liver functions with no evidence of viral markers of hepatitis B or C. Serum levels of tumor markers such as carcinoembryonic antigen (CEA), carbohydrate antigen 19-9 (CA19-9), alpha-fetoprotein (AFP), and protein induced by vitamin K absence or antagonist-II (PIVKA-II) were within normal limits. The serum 5-hydroxyindole-acetic acid (5-HIAA) concentration and urinary 5-HIAA excretion were within the normal range. Contrast-enhanced computed tomography (CT) showed a large mass, measuring approximately $15 \mathrm{~cm}$ in diameter, in the right hepatic lobe (fig. 1a). T1-weighted magnetic resonance imaging (MRI) showed a large mass of low intensity, and T2-weighted MRI showed a large mass of high intensity. [18F] fluoro-2-deoxy-D-glucosepositron emission tomography (FDG-PET) increased 18F-FDG uptake (SUVmax $=4.36)$ near the lateral side of a cystic tumor in the right lobe. No FDG-PET-positive mass was detected, other than the liver tumor (fig. 1b).

Furthermore, we evaluated the use of $99 \mathrm{mTc}$-GSA scintigraphy for the assessment of liver functions. The receptor volume of the whole liver (Rmax) was $1.043 \mathrm{mg} / \mathrm{min}$. The receptor volumes of the right and left lobes were $0.1 \mathrm{mg} / \mathrm{min}$ and $0.943 \mathrm{mg} / \mathrm{min}$, respectively. The right lobe of the liver functioned little. Gastrointestinal fiber and colonoscopy showed no findings. No lesions other than the liver tumors were detected.

The preoperative diagnosis was hepatobiliary cystadenocarcinoma, and a right extended lobectomy was carried out. There was no invasion of other organs. The resected specimen weighed $3,400 \mathrm{~g}$. Sectioning of the specimen demonstrated a solid mass with a giant cystic lesion measuring $12 \mathrm{~cm} \times 6 \mathrm{~cm}$ (fig. 2A). Microscopically, the tumor cells showed round and uniform nuclei, and formed solid or trabecular structures. There were few mitotic or necrotic cells in the solid tumor. There was no vascular invasion. Most of the cells in the solid tumor had retention in the cytoplasm resembling plasma cells. Staining by periodic acid-Schiff (PAS) base was negative in the retention.

Immunohistochemical examination revealed that the tumor cells stained positive with antichromogranin A antibody, anti-neuron specific enolase (NSE) antibody, anti-CD56 antibody, and antiS-100 protein antibody (fig. $2 \mathrm{~B}$ ). The tumor cells were not immunoreactive to anti-AFP antibody, or anti-hepatocyte antibody. Most tumor cells were not positive for Grimelius staining; however, some cells infiltrating the cystic wall were positive. In the tumor cells forming a trabecular arrangement, 10$20 \%$ nuclear reactivity for Ki-67 was present. The tumor cells showed papillary infiltration of the cystic lesion. The patient was discharged 14 days after the operation. The patient was followed by our hospital, and he has not been diagnosed for recurrence at present.

\section{Discussion}

Carcinoid tumors are defined as of neuroendocrine origin and have been classified into 3 types: neuroendocrine carcinomas, typical and atypical carcinoids. Neuroendocrine carcinoma is a malignant epithelial neoplasm with high mitotic activity, high nucleoplasmic ratio, necrosis, vascular invasion, high rate of metastasis, and poor prognosis [2]. The typical carcinoid tumor is a low-grade malignant tumor with rare mitotic activity, rare necrosis, monotony of nuclear size and shape, slow growth, and good prognosis. The atypical carcinoid tumor is an intermediate-grade neoplasm generally with punctuated necrosis and intermediate-mitotic activity [3]. The PHCT, in our case, was diagnosed as a typical carcinoid tumor because of the histological findings. It is not characteristic to be shown by radiological findings such as CT, MRI, and FDG-PET. 
Although the tumor showed increased FDG uptake, this is uncommon in carcinoid tumors.

Carcinoid tumors occur commonly in the gastrointestinal tract, and the liver is the most common site for metastasis. A small occult primary carcinoid tumor may be associated with extensive hepatic involvement. The diagnosis of a PHCT is usually difficult, especially in the absence of typical carcinoid syndrome manifestations, such as flushing and diarrhea, and a lack of specific radiological characteristics.

Consequently, careful preoperative and postoperative examinations are necessary to rule out the presence of other origins. We evaluated the liver function of the patient by 99mTc-GSA scintigraphy. Technetium-99m-diethylene triamine penta-acetic acidgalactosyl human serum albumin (Tc-GSA) is a liver scintigraphy which binds to the asialoglycoprotein receptors on hepatocytes. When we perform hepatectomy, we decide on the extent of liver resection by this scintigraphy and prevent liver failure.

The origin of PHCT remains unknown. Various reports have suggested that it may originate from transformation of liver stem cells [4], or the proliferation of neuroendocrine cells from the bile ducts [5]. PHCTs are very rare and only 90 cases have been reported worldwide. PHCT was found not only in unilobar or bilobar circumstances, but also as a single tumor or multiple tumors. PHCT often shows cystic lesion with solid tumor.

Radiologically, most PHCTs were reported as hypervascular tumors. The treatment of PHCTs depend on the resectability. When the tumor is respectable, surgical operation is considered with long-term tumor free survival. In unresectable cases, arterial chemoembolization, or administration of octreotide acetate or streptozocin, or liver transplantation provide favorable responses. Liver transplantation is a well-recognized option for some neuroendocrine tumors of the liver. Indeed, we performed complete resection of the tumor. In reviews, studies showed that the resectability of PHCT was 70\% and the tumor recurrence was $18 \%$ after 5 years with a 5-year survival of 74-90\% [6].

The diagnosis of the PHCT was difficult because the liver is a common site of metastasis of carcinoid tumors occurring in other organs. Active exclusion of an extrahepatic primary site is essential for the diagnosis of PHCT. Histological study was the only way to obtain a final diagnosis. PHCTs have neuroendocrine differentiation and classical architecture of trabecular, insular, or ribbon-like cell clusters with abundant vascularity of the stroma, uniform cell size with generally rounded nuclei, and no evidence of tumor necrosis. Immunohistochemically, chromogranin-A, NSE, and synaptophysin can be used to confirm the diagnosis of a neuroendocrine tumor such as a PHCT. We diagnosed the tumor as a PHCT because of histologic and immunohistochemical findings $[7,8]$. In addition, preoperative examination and intraoperative observation revealed no other primary lesion. These are useful points to diagnose a liver tumor as a PHCT.

In conclusion, we performed surgical treatment of the PHCT which can be considered successful at present. However, a long-term follow-up is required to exclude occult extrahepatic primaries because carcinoid tumors have slow progressive growth. 
Fig. 1. a Abdominal CT revealed a large mass in the right lobe of the liver. b FDG-PET revealed the uptake lesion near the lateral side of the cystic tumor.

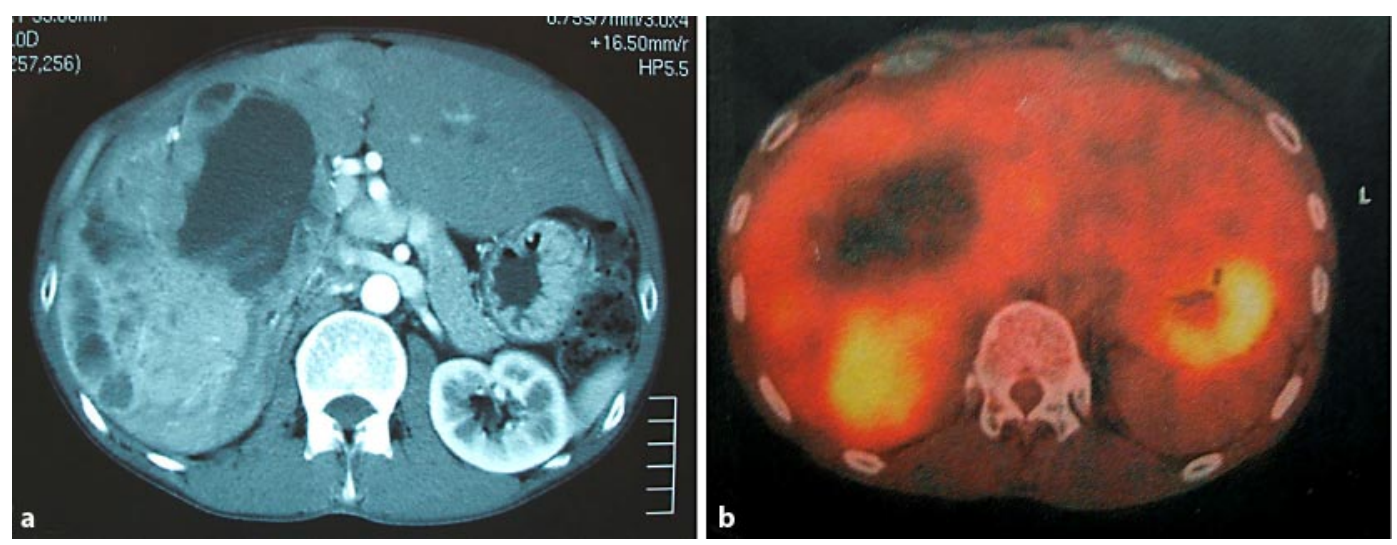




\begin{tabular}{r|l|l|l} 
Case Reports in & $\begin{array}{l}\text { Case Rep Gastroenterol 2009;3:30-35 } \\
\text { D01: 10.1159/000208375 }\end{array}$ & Published online: April 7, 2009 & $\begin{array}{l}\text { O 2009 S. Karger AG, Basel } \\
\text { ISSN 1662-0631 } \\
\text { www.karger.com/crg }\end{array}$ \\
\hline
\end{tabular}

Fig. 2. A The cut surface of the specimen showed a cystic mass with a solid mass surrounding the cystic lesion (arrows). B Immunohistochemical findings showed tumor cells stained positive with a chromogranin A stain, $\mathbf{b}$ anti-NSE antibody, $\mathbf{c}$ anti-CD56 antibody, and $\mathbf{d}$ anti-S-100 protein.

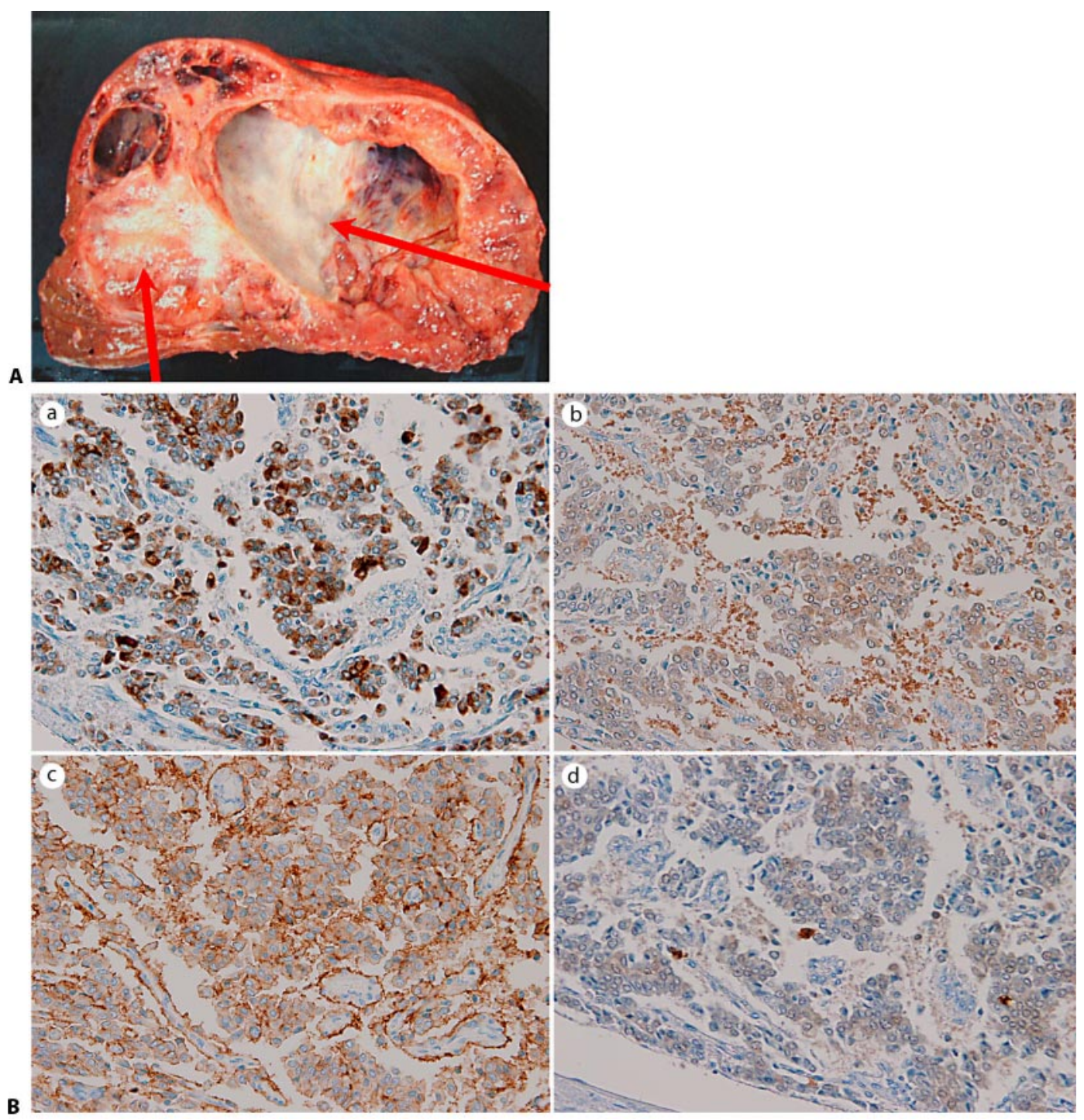




\section{References}

1 Oberndorfer S: Karzinoide Tumoren des Dünndarms. Frankf Z Pathol 1907;1:426-429.

2 Iimuro Y, Deguchi Y, Ueda Y, Tanaka A, Iwasa Y, Ishihara M, et al: Primary hepatic carcinoid tumor with metachronous lymph node metastasis after longterm follow up. J Gastroenterol Hepatol 2002;17:1119-1124.

-3 Staren ED, Gould EV, Warren WH, Wool NL, Bines S, Baker J, et al: Neuroendocrine carcinomas of the colon and rectum: a clinicopathologic evaluation. Surgery 1988;104:1080-1089.

4 Barsky SH, Linnoila I, Triche TJ, Costa J: Hepatocellular carcinoma with carcinoid features. Hum Pathol 1984;15:892-894.

5 Alpert LI, Zak FG, Werthamer S, Bochetto JF: Cholangiocarcinoma: a clinicopathologic study of five cases with ultrastructural observations. Hum Pathol 1974;5:709-728.

-6 Iwao M, Nakamuta M, Enjoji M, Kubo H, Fukutomi T, Tanabe Y, et al: Primary hepatic carcinoid tumor: case report and review of 53 cases. Med Sci Monit 2001;7:746-750.

7 Le Treut YP, Delpero JR, Dousset B, et al: Results of liver transplantation in the treatment of metastatic neuroendocrine tumors. Ann Surg 1997;225:355-364.

8 Lehnert T: Liver transplantation for metastatic neuroendocrine carcinoma: an analysis of 103 patients. Transplantation 1998;66:1307-1312. 